\title{
Index
}

Afghanistan, invasion of 20-1, 46, 88

Alajbegović, Zemira 96, 110 see also Borghesia

Albania 21

Amnesty International 135

Anđelkovic, Zoran 164, 172, 193n.15 anti-bureaucratic revolution 160, 167

see also Milošević, Slobodan antifascism see Second World War anti-semitism 108

Article 133 see verbal crime

Association for a Yugoslav democratic initiative see UJDI

Avdić, Senad 61, 101, 144, 170-2

Berlin 132, 135, 152n.23

Berlin Wall 178, 202

Blazević, Dunja 55

Bogićević, Bogić 48

Bohn, Chris 128

Borghesia 96, 110, 129, 131, 202

Botteri, Robert 70-1, 73, 94, 108, 111 , 147,149

Bulatović, Momir 194n.27

C̆ecko, Ivan 157n.81

Chernobyl 127, 138-9

Chomsky, Noam 42

citizenship 22, 28, 76, 90 acts of 12-13, 126, 150, 198, 200

(multi)layered 6, 92-4, 161, 188-9, 201

see also Yugoslavism civil society $11-12$, 125-6

see also dissidence

conceptual art 56, 63, 66, 122n.48

conscientious objection 126-7, 138, 140, 144,172

(de)criminalisation 142, 146-9

see also military service

constitution

amendments 17, 137, 163, 167-8, 193n.26, 194n.40, 200

federal 1, 23, 57, 65-6, 142, 145, 149, 166

Ćurgus, Velimir Kazimir 40, 63

Czechoslovakia 59, 72, 129, 188

Debeljak, Aleš 130

decentralisation 14, 27, 37, 52-3, 57, 59, 72, 85n.103, 141, 159, 181

dissidence 3, 11-12, 129, 137, 150-1 see also civil society

Dolar, Mladen 107

Đukanović, Milo 194n.27

East Germany 11, 13, 76, 145

economy 8, 18-19, 21-3, 39, 47-8, 65, 74, 76-7, 90, 127, 164-5, 167, 185, 192n.6, 196n.62

see also self-management; unemployment education 1, 21, 25, 129, 154n.43

military 140-1, 155n.56, 156n.60

environmentalism see nuclear

disarmament 
Europe

Eastern 5, 9, 63, 73, 127-8, 136, 152n.27, 164

EC 18, 72, 127, 188

European Community see EC

'European declaration' 176

Europeanisation/integration 72, 167, 179, 185

(West) European belonging 6, 26, 127-32 see also internationalism

feminism 133-4, 136-7, 152n.27

FV 112/15 57, 82n.65, 96, 122n.57

see also Alajbegović, Zemira; Borghesia

gay/lesbian activism see homosexuality

GDR see East Germany

Gojković, Srđan-Gile 56, 59, 113, 115

Gotovac, Tomislav 57, 66-7

Great Britain 68, 83n.80, 108, 128-9, 133, 152n.27

England 94, 132, 153n.29

Grebo, Zdravko 177, 195n.54

Greganović, Branko 174

Helsinki Watch 148, 150

Hemon, Aleksandar 112

homosexuality 70, 131-8

(de)criminalisation 153n.29

film festivals 135, 153n.36

in popular culture 153n.29

Hren, Marko 130, 143, 148

human rights $71,116,129,137,147-8$, 154n.43, 161, 165-6, 176, 182, 193n.22

freedom of expression 43, 54, 65-70, 84n.88, 96, 103-4, 112, 118, 125, 166, 199

see also media

internationalism 1, 5-6, 40, 43, 46, 55, 94, 127-8

see also Europe

Ivančić, Aldo 202

Ivanov, Risto 98, 168, 183-4

Janjatović, Petar 50-1, 54, 58, 62-3, 171

Janša, Janez 149, 158n.85, 192, 194n.40
Jergovic, Miljenko 191

JNA 88, 140-7, 155nn.56-7, 156n.62, 157n.68, 169-70, 180, 182, 190, 192

see also military service

Jović, Dejan 52, 73, 94, 106, 139, 159, 170, 183

FUPIO 17, 24, 46, 77, 90-1, 101, 119n.11, 120n. 18

Kadić, Rasim 169, 177, 179-80, 184, 187, 189

Kardelj, Edvard 125, 146, 162

Kontić, Boro 64, 67, 69

Korda, Neven 129

Kosovo 8, 18, 21-2, 24, 53, 73, 120n.13, 126, 133, 164-5, 167-8, 171, 176, 193n.26, 197n.73, 200

Kostov, Zoran 179, 184

Kovač, Miha 68, 70, 165, 170, 178

Kremer, Dragan 58-9, 67, 98-9, 171

Kučan, Milan 8, 159, 162

Laibach 13, 64, 88, 107-9, 112, 122n.51

League of Communist Youth of Yugoslavia see SSOJ, SKOJ

League of Communists of Yugoslavia see SKJ

League of Socialist Youth of Bosnia see SSOJ, SSO Bosnia

League of Socialist Youth of Croatia see SSOJ, SSO Croatia

League of Socialist Youth of Macedonia see SSOJ, SSMM

League of Socialist Youth of Serbia see SSOJ, SSO Serbia

League of Socialist Youth of Slovenia see SSOJ, ZSMS

League of Socialist Youth of Yugoslavia see $\mathrm{SSOJ}$

liberalisation 12, 52, 63, 72, 89, 135, 161

liberalism 45, 49, 72-3, 146, 159-60, 168, 176, 183, 186, 192n.6, 201

liberal parties 164, 184-5, 193n.21, 196n.62

Lončar, Budimir 179

Lumumba, Patrice 40, 75 
Mamula, Branko 74, 76, 86n.121, 105, 190

Mandić, Oliver 153n.29

Mannheim, Karl 7, 9, 24

Marković, Ante 174-6, 179, 185-7

Marxism 1, 9, 40, 49, 59, 78, 94, 138, 146,159

MASPOK 45

Mastnak, Tomaž 124, 129

media 23, 68, 72, 85n.103

bans $60,69,71,73-5,86 n .119$

legal framework 66, 84n.88

youth print media 53-4, 61

Mlad borec 67, 174-5

Mladina 68, 70, 71, 74, 76, 85n.106, 101-2, 111, 135, 141, 147, 149, 15ln.11, 165, 191, 203

Mladost 26, 61, 76, 146, 170, 172

Naši dani 54, 76, 86n.119, 171,175

Polet 59, 66, 73, 76, 132, 174

Student 43, 171-2

Studentski list 74-5, 139

youth radio 54,59

in Belgrade 62, 171

in Ljubljana 68-70, 117, 127-8

Sarajevo youth program 64,68

in Zagreb 57, 82n.70

Western 23, 65, 186

Mijanović, Vladimir 42

Mijović, Vlastimir 172

military service 19, 125, 141-2, 145

legal amendments 144, 148

in Penal code 156n.63

see also conscientious objection

Milošević, Slobodan 8, 74, 95, 166-7, 169-72, 174, 178-9, 190, 201

Minić, Miloš 42

Mladenov, Nikola 175, 195n.47

Mlađenović, Lepa 136

Mladenović, Milan 198

Najdovski, Slobodan 184

nationalism 18, 43, 50, 72, 94-5, 116, 118 , $136,166,168-71,173,175,181$, 190, 201-2

Nazism 43, 103, 107-8

'the Nazi party' 110-11, 191
Nazi-punk affair 116-18

Riefenstahl, Leni 110

see also Laibach

new social movements 18, 28, 51, 125-7, 131, 133, 135-6, 139-40, 146, 150, 163, 185

see also conscientious objection; homosexuality; nuclear disarmament

new wave $10,26,51,56,62-3,113$, 122n.51, 153n.29

see also punk

non-alignment 20, 46, 113, 128, 182

nostalgia 106, 129

Nova revija 95, 170, 185

NSK (Neue Slovenische Kunst) see Laibach

nuclear disarmament 28, 125-7, 138-9, 150, 154n.50, 155n.52

OOUR (Basic organisation of associated labour) see self-management

Ordanoski, Sašo 67, 92

Pavićević, Borka 40, 42

Pećanin, Senad 54, 64, 67, 77, 96, 111,177

Poland 21, 23, 127-8, 132, 148, 151n.1, 152n. 27

Solidarity 21, 124, 151n.4, 164

Popit, Franc 117, 161

Popov, Nebojša 17

'poster affair' 102-4, 116, 199

Predin, Zoran 26, 60-1, 143

protests

in 1968 40, 42-3, 65

in 198121

after Chernobyl 139

freedom of the press 175

'Slovenian spring' 149

strikes after 1985 86n.122, 166-7, 171

punk

British 116, 118, 155

Yugoslav 51, 58-60, 63, 81, 94, 112-13, 115-17, 128

see also new wave

Raguž, Martin 186

Rexhepi, Hashim 22, 73, 126 
Ristić, Ljubiša 42

rock music see new wave; punk

\section{Second World War}

history/legacy 23, 104, 107-8, 120n.22, 146, 188

Partisan generation 7-8, 39, 42-3, 89 youth 27

self-management

associated labour 57, 78n.3, 82n.70

delegate system 19-20, 23, 38, 46, 78n.3, 80n.35, 106, 144-5, $163,168-9$

'pluralism of self-managing interests' 29, 125-6, 147, 161, 166, 180, 182,190

Shala, Blerim 176

SKJ

demise 159, 169, 174, 195n.43

factionalism 69, 72-3, 76, 85n.102, 166

membership 20, 22, 38, 45, 51, 95, 120n. 18, 140-1

reform 8, 161-2, 171, 178, 181-2

youth policy 44-6, 48, 59, 64, 110-11, 116-17

Sklevicky, Lydia 137

Školč, Jozef 183, 194n.40

Socialist Alliance of the Working People of Yugoslavia see SSRNJ

socialist patriotism 1, 38, 45, 104-5

Soviet Union 13, 20-1, 25, 29n.2, 33n.44, 40, 112, 122n.48, 122n.51, 129-30, 133

\section{SSOJ}

congresses 27, 44, 48, 61-2, 73, 109, 112 , 125-6, 139-40, 147, 161-5, 182-4, 187, 192n.3, 196n.66, 197n.73

dissolution 173, 179-87, 196n.62, 196n.66

reform 41, 51-2, 101, 147, 160, $163-6,179-82$

SKOJ 60-1, 78n.4

SSMM 100, 163, 179-80, 183-5

SSO Bosnia 47, 49, 99, 101-2, 169, 173, 177-8, 180-1, 185-6

SSO Croatia 54-5, 60, 73, 75, 81n.53, 133-4, 139, 180-1, 183, 196n.66

SSO Serbia 49, 54, 62-3, 105, 160, 164, 167, 172, 180, 182, 190, 193n.15, 196n.62

SSOJ in the JNA 105, 140, 145, 180, 182, 190

structure 2

ZSMS 73, 102-4, 109, 117, 126, 140-1, 144-7, 150, 161, 164, 174, 176, 180, 183-4, 190, 192n.3, 194n.40

see also Youth Day

SSRNJ 38, 46, 62, 89, 97, 145, 178, 182

Stefanovski, Vlatko 60-1, 128-9

students 20-1, 28, 41-3, 45-6, 50-1, 55-8, 74, 91, 99, 111, 126, $144,171,185$

see also universities

Sukič, Nataša 106, 131, 135-6

supranationalism see Yugoslavism

Surroi, Veton 176

Šuvar, Stipe 73-4, 85n.102, 171

Svilanović, Goran 166, 193n.21

Switzerland 131, 152n.22

Thaler, Zoran 164, 176, 179, 184-5, 188

Tito, Josip Broz 8, 20-1, 72, 75, 108 and 196842

birthday 51, 88, 97, 101, 104, 106

see also Youth Day

cult 28, 38, 88-9, 100, 104, 113 , 130,163

death $21,23,28,45,77,116,118$, 143,199

successors 8, 23, 48

Tomašević, Stana-Arnesen 89

Tomc, Gregor 25, 60, 95, 114-16

Top Lista Nadrealista 64, 83n.81, 102

Trajkovska, Olivera 183

Tratnik, Suzana 131, 136

trials 21, 70, 116, 149, 193n.22

Tupurkovski, Vasil 27, 49-50, 184

UJDI 69-70, 176-8, 187

UN see United Nations

unemployment 18, 42, 47-8, 50, 90, 163 see also economy

United Kingdom see Great Britain

United Nations 147, 166

UNESCO 6, 18, 46, 48, 53, 124, 138

universities 21-2, 41-2, 44-5, 50, 53, 55, $66,74-5,101,141,171$

see also students 
urban-rural divide 13, 26, 43, 45, 91, 113, 174

USSR see Soviet Union

verbal crime 66, 70, 73, 84n.89, 112, 116, 139, 166, 185, 199

Vidmar, Igor 49, 51, 103, 109, 111-12, 116-18, 127-8

Vllasi, Adem 8, 22, 45, 176, 197n.73

voluntary work see youth work actions

Youth Baton 70, 103-6, 111, 159

see also Youth Day

youth cultural centres 21, 41, 55-8, 74, $81 n .55,115,132-3,135,152$ n.27
Youth Day 16, 88-9, 97, 101-6, 110, 180, 199

see also Tito, Josip Broz; Youth Baton youth work actions 90, 97-100, 106

Yugoslav People's Army see JNA

Yugoslav program for youth research see FUPIO

Yugoslavism 5-6, 22, 91-5, 113, 174, 183, 188-9, 191, 198, 201

see also socialist patriotism

Zabranjeno pušenje 59, 72, 83n.81, 102

Živadinov, Dragan 147

Žugić, Silvija-Rijavec 162 
Ljubica Spaskovska - 9781526106339 Downloaded from manchesterhive.com at 04/26/2023 09:07:57AM via free access 
Ljubica Spaskovska - 9781526106339 Downloaded from manchesterhive.com at 04/26/2023 09:07:57AM via free access 
Ljubica Spaskovska - 9781526106339 Downloaded from manchesterhive.com at 04/26/2023 09:07:57AM via free access 
Ljubica Spaskovska - 9781526106339 Downloaded from manchesterhive.com at 04/26/2023 09:07:57AM via free access 
Ljubica Spaskovska - 9781526106339 Downloaded from manchesterhive.com at 04/26/2023 09:07:57AM via free access 
Ljubica Spaskovska - 9781526106339 Downloaded from manchesterhive.com at 04/26/2023 09:07:57AM via free access 